\title{
Shuttlebox performance in BALB/cByJ, C57BL/6ByJ, and CXB recombinant inbred mice: Environmental and genetic determinants and constraints
}

\author{
DUDLEY F. PEELER \\ University of Mississippi Medical Center, Jackson, Mississippi
}

\begin{abstract}
Male mice from nine strains (the CXB recombinant inbred set and their progenitors, BALB/cByJ


solve discrepancies between earlier studies. Mice were tested at one of three times of day (TOD) using one of three apparatus configurations. Analysis of variance revealed complex interactions between strain, apparatus configuration, and TOD. Subsequent analyses and tests of simple effects indicated very strong influences of apparatus configuration and/or TOD upon rate of acquisition in some strains, but not in others. C57BL/6 mice were relatively little affected by either variable; BALB/c mice were responsive to apparatus configuration but not to TOD. The recombinant inbred strains performed variously at levels that were either intermediate to, comparable with, or higher than those of the progenitors, depending upon the context of the testing situation. Genetic determination of behavior is, in part, a function of the particular context in which the behavior occurs. Both TOD and apparatus configuration are significant environmental factors.
\end{abstract}

Learned avoidance of footshock in a shuttlebox has variously been reported as reflecting a single major gene effect (Oliverio, Eleftheriou, \& Bailey, 1973) and as being polygenically determined (Peeler, 1987). These two studies utilized the same genetically defined strains, but provided partially contradictory results. However, the differences might in part be explained by differences in some aspects of the testing conditions and procedures used. For example, although dimensions of the test apparatus were very similar, the barriers used were quite different, as were the number of trials per session, the total number of sessions, and the durations of conditional stimulus (CS), unconditional stimulus (US), and intertrial intervals (ITI). Efforts to reduce this discrepancy by closer (but not exact) replication of the techniques used by Oliverio et al. (1973), however, produced results discordant with both of the earlier studies (Peeler, 1988).

Perusal of the data and experimental conditions of the studies revealed that in the case of my own data collection (Peeler, 1987, 1988), no consideration had been given to the possible effects of the time of day upon performance. In the report by Oliverio et al. (1973), there is similarly no indication of the time of day at which testing was conducted. Circadian effects upon behavior in mice are well documented, and the time of day at which activity is measured is known to be a relevant factor in at least some strains (Beau, 1991; Hostetter, 1966; Lassalle

Correspondence should be addressed to D. F. Peeler, Department of Neurosurgery, University Medical Center, $2500 \mathrm{~N}$. State Street, Jackson, MS 39216 (e-mail: dfp@anat.umsmed.edu).
\& Le Pape, 1978; Oliverio \& Malorni, 1979; Peeler, 1990; Poirel, 1988; Possidente \& Birnbaum, 1979; Schwartz \& Zimmerman, 1990; Vitaterna et al., 1994). Apparatus configuration also may influence activity (Peeler, 1989, 1995). The investigations reported herein were aimed at resolving the discrepancy in results by examining the effects of time of day of testing and different apparatus configurations upon shuttlebox avoidance.

The CXB set of recombinant inbred mouse strains is dichotomous for an apparently monogenically determined aberration in hippocampal structure, the hippocampal lamination defect (Hld; Nowakowski, 1984). Although the data from Oliverio et al. (1973) and Peeler (1987) do not indicate any association between Hld and shuttlebox avoidance, the variability in strain-distribution patterns noted above indicated that further investigation was warranted. The present study provides an opportunity to reassess possible effects of Hld.

\section{METHOD}

\section{Subjects}

The subjects were 532 adult male mice from nine strains: the progenitor strains $\mathrm{C} 57 \mathrm{BL} / 6 \mathrm{ByJ}$ and $\mathrm{BALB} / \mathrm{cByJ}$, and the seven recombinant inbred (RI) strains derived from them-namely, $\mathrm{CXBD} /$ ByJ, CXBE/ByJ, CXBG/ByJ, CXBH/ByJ, CXBI/ByJ, CXBJ/ByJ, and $C X B K / B y J$. The animals were obtained from the breeding colony in the neurosurgery laboratory at the University of Mississippi Medical Center. (This colony has been maintained through intrastrain breeding since 1986; the mice originally were obtained from Jackson Laboratory, Bar Harbor, Maine, and supplementation with additional breeders has been rarely necessary, the last such occasion being March, 1992.) All but a few mice were 85-95 days old when first tested; the extremes were 70-120 days old. The subjects 
were housed in group cages in a room with controlled temperature, humidity, and lighting. Room lights were on for $12 \mathrm{~h}$ each day (06:00-18:00).

\begin{abstract}
Apparatus
A clear plastic box $(12.5 \times 30.2 \times 14 \mathrm{~cm})$ with a floor of alternately wired steel bars was used for avoidance training. The bars were connected to an alternating $(60-\mathrm{Hz})$ current source (current was provided through a high-voltage transformer [800v] and a high resistance that could be adjusted between 1.3 and 1.5 megohm). Current levels, as read from a meter in series with the subject, varied between 0.4 and $0.8 \mathrm{~mA}$. The box was divided at the middle by one of three barriers, described below. Shock was applied to only one half of the floor (as partitioned by the barrier) at any given time. Single lights mounted over each half of the box indicated the area that would receive shock current. Incidental auditory cues were provided by relays controlling onset and cessation of the CS and US.
\end{abstract}

\section{Procedure}

Mice were tested for acquisition of shuttlebox avoidance in the following three sequential independent phases that employed identical procedures and apparatus, except for the configuration of the barrier separating the two halves of the shuttlebox: Phase 1: the hurdle, a $2.5-\mathrm{cm}$-high translucent plastic strip extending across the width of the box; Phase 2: the hole, an opaque divider extending the full height and width of the box, with a $4 \times 6-\mathrm{cm}$ rectangular opening extending upward from a point $2 \mathrm{~cm}$ above the floor; and Phase 3: the slot, a barrier similar to the hole partition, but with a $4 \times 8-\mathrm{cm}$ opening extending upward from the floor. The hurdle barrier was the same as that used by Peeler (1987); the hole barrier essentially replicated the barrier used by Oliverio et al. (1973), as described in Bovet, Bovet-Nitti, \& Oliverio (1966, 1969); and the slot barrier was an attempt to eliminate the apparent obstruction to crossing caused by the lower border of the hole barrier. There were 178 subjects in Phase 1, 182 subjects in Phase 2, and 172 subjects in Phase 3. On average, the groups contained 6 individuals each, with a range of 5-10 individuals.
A trial consisted of a maximum of $10 \mathrm{sec}$ of light $(\sim 3.7 \mathrm{~lx})$ centered over the side in which the mouse was located. During the first $4 \mathrm{sec}$, no shock was applied to the floor. During the last $6 \mathrm{sec}$, electric current $(\sim .75 \mathrm{~mA})$ was applied to the floor of the lighted half of the box. Each trial was terminated when the subject crossed the barrier or at the end of $10 \mathrm{sec}$. Crossing the barrier during the first $4 \mathrm{sec}$ of a trial constituted an avoidance; crossing during the last $6 \mathrm{sec}$ was an escape response. The trials were separated by an interval of $16 \mathrm{sec}$. No shock was applied to the floor in either half of the box during the ITI. Each subject was exposed to this procedure for 50 trials per day over five consecutive days at one of the following three times of day (TOD): 07:00-09:00 (TOD 1); 10:00-12:00 (TOD 2); or 13:00-15:00 (TOD 3). These times for testing were chosen on the basis of previously obtained data concerning variation in activity during these periods in these same strains (Peeler, 1990). Testing was conducted in a darkened room, with a dim light $(\sim 0.02 \mathrm{~lx})$ provided through a diffuser behind the shuttlebox.

\section{RESULTS}

The number of avoidance responses in each session by each subject served as the data. A probability of $\alpha=.05$ or less was accepted as statistically significant. An initial analysis of variance (ANOVA), encompassing the between-subjects dimension of strain, partition, and TOD and the within-subjects dimension of sessions, revealed a significant interaction between the four factors $[F(128,1804)=1.677, p<.0001]$. Strain $[F(8,451)=$ $33.269, p<.0001]$ and partition $[F(2,451)=83.794, p<$ $.0001]$ were significant main effects; TOD was not. All of the first- and second-order interactions, except that of sessions with TOD, were significant.

For purposes of comparison with other studies (which generally have not been concerned with interactions), it

Table 1

Strain Distributions from Present and Previous Studies








Figure 1. Distribution patterns within and among strains of mean number of avoidance responses for 250 trials. Bars indicate standard error for each group.

is appropriate to consider the scores for strains pooled over all conditions. Thus, ignoring the effects of the different apparatus configurations, TODs, and rates of acquisition (i.e., considering only the significant strain effect), the strain distribution shown in Table 1 is obtained (in these and all subsequent comparisons, strains not underlined in common are statistically significantly different $[p \leq .05]$ ). However, as may be seen in Figure 1 (which presents the strain $\times$ partition interaction), although some strains tend to maintain relative position in the distribution when apparatus configuration is altered, others do not. (The statistical differences are shown in the lower part of Figure 1.) This strain $\times$ partition interaction reflects, in some cases (e.g., CXBD), a detrimental effect of changing from hurdle to hole to slot; in others (e.g., $\mathrm{C} 57 \mathrm{BL} / 6$ and $\mathrm{CXBH}$ ), configurational change is apparently irrelevant. Similarly, the TOD and sessions dimensions have different effects for different strains. Table 2 shows the mean number of avoidance responses for each strain at each TOD under each configuration. An overview of the interactive relationship of strain, TOD, and session for each partition is presented in Figures 2, 3, and 4.

All strains show statistically significant increases in the number of avoidances over the five sessions, but in some strains, as may be seen in Figures 2, 3, and 4, apparatus configuration and TOD substantially influence the shape and height of avoidance-acquisition curves. For the hurdle and hole partitions, the interaction between session, strain, and TOD is statistically significant
$[F(64,604)=2.202, p<.0001$, and $F(64,620)=1.876$, $p<.0001$, respectively]; for the slot partition, the only significant interaction is that of strain $\times$ sessions $[F(64,580)=7.973, p<.0001]$. Strain is a significant main effect in all three partitions $[F(8,604)=14.713, p<$ $.0001, F(8,620)=10.587, p<.0001$, and $F(8,580)=$ $22.732, p<.0001$, respectively], while TOD is a significant main effect $[F(2,620)=3.263, p=.0409]$ only for the hole partition. Subsequent ANOVAs and tests of simple effects (Newman-Keuls) indicate that strain is a significant factor at each TOD during Sessions 2, 3, 4, and 5 for both hurdle and hole partitions (for hurdle, Session 5, TOD $2, p=.0562$ ). The statistical relationships among strains are shown in Figure 5.

\section{Between-Strain Effects}

Hurdle partition. Strains CXBD and CXBH are significantly superior to the other strains in avoidance during TOD 1, Session 2 (see Figure 5). However, these statistical distinctions become less clear in Sessions 3 and 4, and are essentially lost by Session 5 (Strain CXBE actually shows regressing avoidance performance during the last two sessions, and its performance is significantly lower than that of all other strains by Session 5). At TOD 2, the distribution of strain means is altered: CXBD tends to be significantly superior to other strains in avoidance (except in Session 3, in which it is significantly superior to all other strains except Strain CXBE). This distinction is less clear in Session 4, and in Session 5, only the extremes- 
Table 2

Mean Number of Avoidances ( $M$ ), Variance, Standard Error $(S E)$, and Number of Mice per Group ( $N$ ) for each Strain at each Time of Day (TOD)

\begin{tabular}{|c|c|c|c|c|c|c|c|c|c|c|c|c|}
\hline \multirow[b]{3}{*}{ TOD } & \multicolumn{12}{|c|}{ Partition } \\
\hline & \multicolumn{4}{|c|}{ Hurdle } & \multicolumn{4}{|c|}{ Hole } & \multicolumn{4}{|c|}{ Slot } \\
\hline & $N$ & $M$ & Variance & $S E$ & $N$ & $M$ & Variance & $S E$ & $N$ & $M$ & Variance & $S E$ \\
\hline \multicolumn{13}{|c|}{$\mathrm{C} 57 \mathrm{BL} / 6$} \\
\hline 1 & 7 & 74.3 & 1408.576 & 14.185 & 8 & 52.1 & 867.008 & 10.410 & 8 & 67.1 & 2392.384 & 17.293 \\
\hline 2 & 7 & 81.0 & 1401.679 & 14.151 & 7 & 90.9 & 5088.540 & 26.962 & 8 & 48.5 & 2342.270 & 17.111 \\
\hline 3 & 7 & 74.9 & 829.152 & 10.883 & 7 & 71.3 & 2276.244 & 18.033 & 6 & 87.0 & 2341.592 & 19.755 \\
\hline \multicolumn{13}{|c|}{ CXBD } \\
\hline 1 & 6 & 165.2 & 2589.385 & 20.774 & 8 & 48.4 & 1979.449 & 15.730 & 6 & 72.8 & 2143.412 & 18.900 \\
\hline 2 & 6 & 167.0 & 167.988 & 5.292 & 6 & 118.8 & 89.359 & 3.859 & 6 & 43.7 & 1337.877 & 14.932 \\
\hline 3 & 6 & 169.7 & 359.482 & 7.740 & 10 & 48.5 & 2181.637 & 14.770 & 7 & 43.7 & 1447.574 & 14.380 \\
\hline \multicolumn{13}{|c|}{ CXBE } \\
\hline 1 & 7 & 67.1 & 1035.810 & 12.164 & 7 & 65.4 & 1301.983 & 13.638 & 6 & 37.8 & 506.160 & 9.185 \\
\hline 2 & 7 & 135.9 & 2092.148 & 17.288 & 6 & 142.3 & 874.267 & 12.071 & 6 & 29.2 & 716.151 & 10.925 \\
\hline 3 & 6 & 165.3 & 301.057 & 7.084 & 6 & 87.5 & 3748.256 & 24.994 & 6 & 40.8 & 773.785 & 11.356 \\
\hline \multicolumn{13}{|c|}{ CXBG } \\
\hline 1 & 7 & 107.1 & 5668.735 & 28.458 & 7 & 30.6 & 3384.563 & 21.989 & 7 & 19.4 & 840.594 & 10.958 \\
\hline 2 & 6 & 111.7 & 3890.641 & 25.465 & 7 & 44.1 & 777.127 & 10.537 & 6 & 22.5 & 445.083 & 8.613 \\
\hline 3 & 6 & 77.8 & 1664.966 & 16.658 & 7 & 46.3 & 1152.263 & 12.830 & 6 & 10.7 & 124.256 & 4.551 \\
\hline \multicolumn{13}{|c|}{$\mathrm{CXBH}$} \\
\hline 1 & 6 & 160.2 & 1008.190 & 12.963 & 6 & 137.7 & 1689.457 & 16.780 & 6 & 156.0 & 1566.814 & 16.160 \\
\hline 2 & 6 & 84.5 & 2037.078 & 18.426 & 6 & 65.3 & 3835.820 & 25.285 & 7 & 135.7 & 1830.556 & 16.171 \\
\hline 3 & 10 & 163.5 & 521.163 & 7.219 & 6 & 160.5 & 631.517 & 10.259 & 7 & 144.7 & 2098.556 & 17.315 \\
\hline \multicolumn{13}{|c|}{ CXBI } \\
\hline 1 & 6 & 100.8 & 715.349 & 10.919 & 6 & 20.5 & 683.509 & 10.673 & 6 & 64.7 & 1029.896 & 13.101 \\
\hline 2 & 6 & 89.3 & 1773.084 & 17.190 & 5 & 18.4 & 479.303 & 9.791 & 5 & 66.6 & 4391.315 & 29.635 \\
\hline 3 & 6 & 113.7 & 503.464 & 9.160 & 7 & 57.9 & 879.123 & 11.207 & 5 & 105.4 & 4834.838 & 31.096 \\
\hline \multicolumn{13}{|c|}{ CXBJ } \\
\hline 1 & 8 & 97.5 & 1774.010 & 14.891 & 7 & 16.1 & 376.127 & 7.330 & 6 & 29.5 & 1461.915 & 15.609 \\
\hline 2 & 6 & 63.3 & 1547.478 & 16.060 & 7 & 29.6 & 1467.963 & 14.481 & 6 & 20.0 & 813.219 & 11.642 \\
\hline 3 & 6 & 95.2 & 496.978 & 9.101 & 6 & 61.7 & 2390.623 & 19.961 & 7 & 9.9 & 143.472 & 4.527 \\
\hline \multicolumn{13}{|c|}{ CXBK } \\
\hline 1 & 6 & 124.8 & 444.156 & 8.604 & 6 & 60.5 & 1412.332 & 15.342 & 6 & 71.0 & 1797.590 & 17.309 \\
\hline 2 & 6 & 111.2 & 3387.007 & 23.759 & 7 & 73.4 & 1433.607 & 14.311 & 6 & 65.5 & 2928.325 & 22.092 \\
\hline 3 & 6 & 78.2 & 2576.984 & 20.724 & 7 & 39.3 & 2543.286 & 19.061 & 9 & 51.3 & 1059.763 & 10.851 \\
\hline \multicolumn{13}{|c|}{$\mathrm{BALB} / \mathrm{c}$} \\
\hline 1 & 8 & 51.9 & 341.843 & 6.537 & 6 & 33.8 & 2202.988 & 19.161 & 6 & 20.8 & 348.569 & 7.622 \\
\hline 2 & 7 & 43.9 & 333.793 & 6.906 & 7 & 44.4 & 1755.275 & 15.835 & 6 & 7.2 & 43.771 & 2.701 \\
\hline 3 & 7 & 76.7 & 971.257 & 11.779 & 7 & 29.3 & 394.578 & 7.508 & 6 & 15.0 & 179.212 & 5.465 \\
\hline
\end{tabular}

CXBD and BALB/c - are significantly different. At TOD 3, Sessions 2, 3, and 4, Strains CXBH, CXBE, and CXBD have significantly more avoidances than all other strains. The differences are not as clear for Session 5, although the distribution of strains remains essentially the same.

Hole partition. The distribution is changed: $\mathrm{CXBH}$ mice are the best performers at TOD 1, having significantly more avoidances overall than all other strains. Again, by Session 5, other strains have improved their performance and statistical distinctions are less clear. At TOD 2, however, $\mathrm{CXBH}$ mice had reduced performance, with CXBE being significantly better than all other strains. Strains CXBD and CXBE tend to be significantly different from Strains CXBI and CXBJ. During TOD 3, the CXBH mice once again had significantly more avoidances overall than all other strains, although in Sessions 4 and
5, CXBJ, C57BL/6, and CXBE mice were no longer different.

Slot partition. With slot as the partition, there was a significant interaction of strain with session, but not of strain with TOD. CXBH mice stand out as having superior performance to that of all other strains.

The progenitor strains, it may be noted, were consistently statistically significantly different only when the configuration that was apparently the most difficult (i.e., the slot partition) was used (in Sessions 3, 4, and 5). Only at TOD 2, Session 2, for hole (in which they were also different) and TOD 3, Session 3 for slot do the RI strains form an unambiguous dichotomy around the two progenitors. Strains CXBD and CXBE segregate with C57BL/6 using the hole partition; Strains $\mathrm{CXBH}$ and $\mathrm{CXBI}$ segregate with $\mathrm{C} 57 \mathrm{BL} / 6$ using the slot configuration. 


\section{Within-Strain Effects}

Viewing the data in terms of the response of each strain to the TOD and partition variables, one strain $-\mathrm{C} 57 \mathrm{BL} / 6$ was essentially impervious to any changes: for this strain, neither partition nor TOD effects were significant, nor was any interaction involving those two variables. In three strains-BALB/c, CXBG, and CXBK- the main effect of partition was statistically significant, without interaction. In five strains (CXBD, CXBE, CXBH, CXBI, and $\mathrm{CXBJ})$, the sessions $\times$ TOD $\times$ partition interaction was significant. (For Strains CXBD, CXBE, CXBI, and CXBJ, partition was also a significant main effect; TOD was a significant main effect for Strains CXBE, CXBI, and $\mathrm{CXBH}$ ).

In all strains in which partition was a significant factor, the performance on the hurdle task was superior to that with the hole or the slot task. For Strains CXBD, CXBE, CXBJ, and CXBK, hurdle performance was superior throughout to either hole or slot performance (these two being essentially equivalent), although all three tasks tended to show improvement over sessions. Strain CXBG began similarly, but by Session 5, hurdle performance was superior to hole performance, which was superior to slot performance. Strain CXBI began with hurdle and slot performance being similar, and with both being superior to hole performance, but in Session 5, hurdle performance was superior to slot performance, which was superior to hole performance. Strain BALB/c showed no effect of partition in the first two sessions. In Session 3, hurdle and hole performance were superior to slot performance; in Session 4, hurdle performance was superior to hole performance, which was superior to slot performance; and in Session 5, hurdle performance was superior to hole and slot performance, which were statistically equivalent.

Incidental observations revealed that the CXBD mice, when confronted with footshock, appeared predominantly to seek escape by moving generally upward, running with heads up, jumping, and attempting to climb the walls. They learned relatively soon to escape quickly and then to avoid footshock, particularly in the hurdle configuration. At the other extreme, in a greater percentage of their responses, BALB/c mice appeared in the present instance to attempt escape predominantly by going in

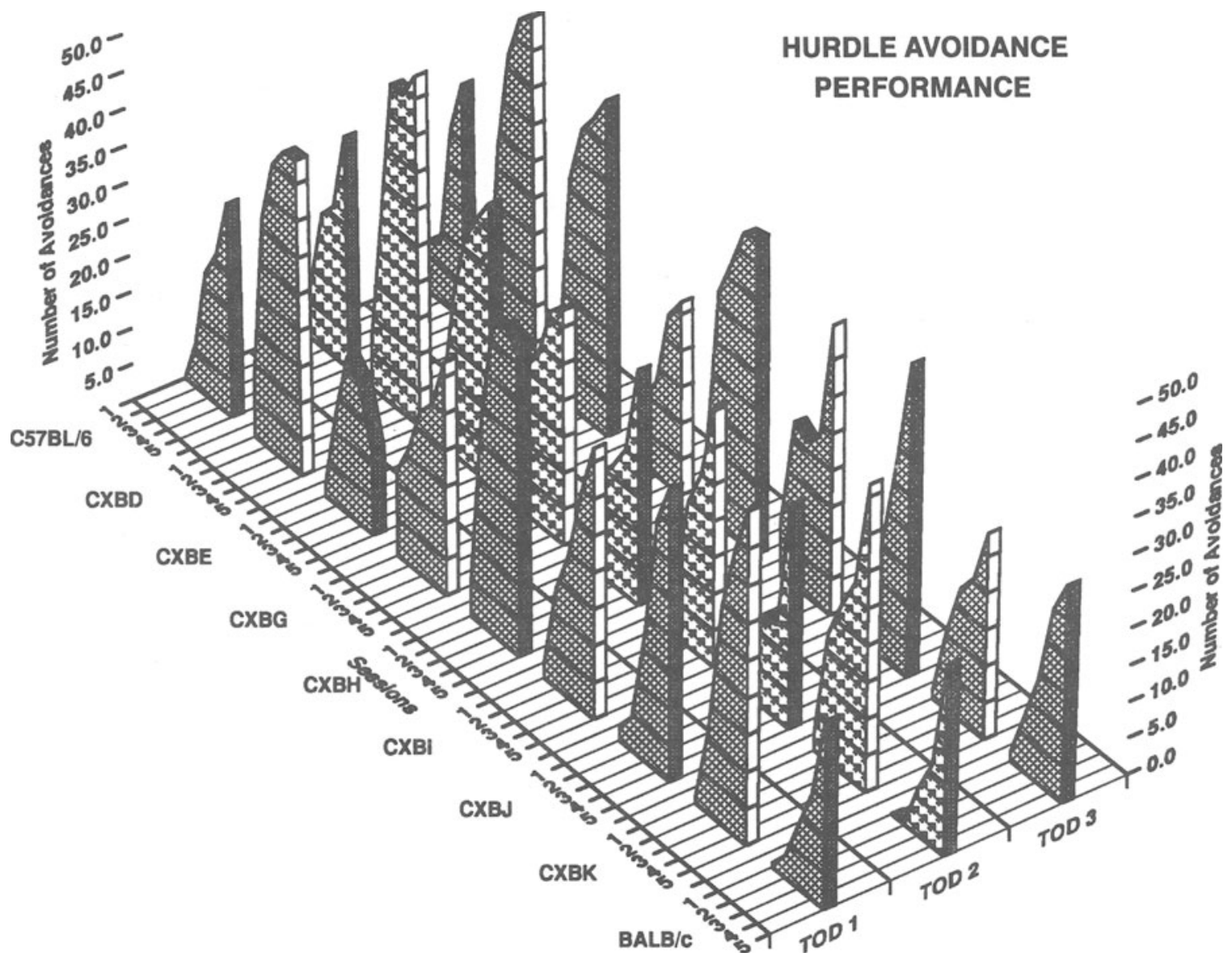

Figure 2. Topography of shuttlebox avoidance-acquisition curves for C57BL/6ByJ, BALB/cByJ, and the CXB set of recombinant inbred strains, with the hurdle partition. The leading (time of day, or TOD) surfaces of the performance curves are alternately darkly shaded or not shaded, to aid in distinguishing strains across the TOD dimension. The TODs are distinguished within strains by shading of the sessions surface. Vertical lines on a curve surface mark sessions; horizontal lines indicate mean number of avoidances, in 5-unit increments. 


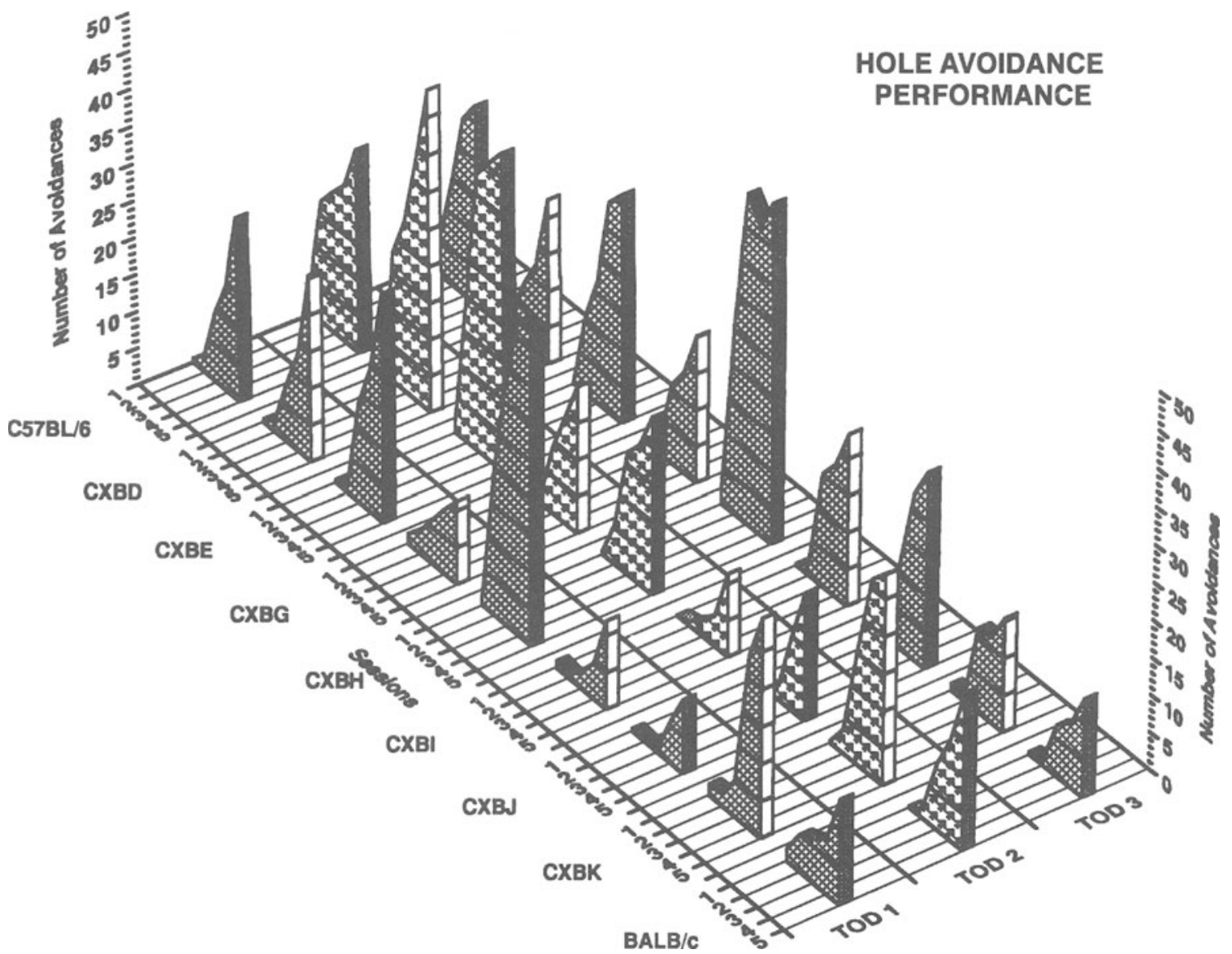

Figure 3. Topography of shuttlebox avoidance-acquisition curves for C57BL/6ByJ, BALB/cByJ, and the CXB set of recombinant inbred strains with the hole partition. The leading (time of day, or TOD) surfaces of the performance curves are alternately darkly shaded or not shaded, to aid in distinguishing strains across the TOD dimension. The TODs are distinguished within strains by shading of the sessions surface. Vertical lines on a curve surface mark sessions; horizontal lines indicate mean number of avoidances, in 5-unit increments.

the other direction; that is, they, too, initially ran about in response to footshock, but frequently with heads lowered and with repeated efforts to get out by going between the bars. With repeated trials, a few quickly learned not to escape, but to hold on and endure. Perhaps because they did not quickly learn to avoid by jumping or climbing, a few other rather innovative individuals did manage in some instances to escape - but rarely avoid-using such experimenter-frustrating techniques as the one-legged stance: front legs against the barrier or a wall and tail and one rear leg held up, leaving only one leg in contact with the electrically conductive bars. Of course, all strains, and all individuals within each strain, engaged in a variety of escape-directed behaviors, and the foregoing descriptions could be applied to many of them at one time or another. Appropriate escape/avoidance responses were eventually acquired to some degree in all strains. Although general tendencies toward certain approaches to solving the avoidance problem appeared present and noticeable, particularly in the strains at either extreme, the observations were not conducted systematically for the total duration of the study, thus precluding statistically meaningful analysis.
In only one distribution did the RI strains form a dichotomy about the progenitor strains: Slot configuration, TOD 2, Session 2. This distribution matches no pattern for known loci, although statistically it does differ with respect to only one strain from that reported by Moisset (1977) for open-field activity (see Table 1). The strain-distribution patterns (SDPs) for the strain main effect and for the slot partition (ignoring TOD and sessions) are quite similar to those for the loci of several cellsurface antigens, DNA sequences, enzymes, and proteins (Table 1), but since CXBH performance is statistically different from that of both progenitor strains, more than one locus is indicated. The SDP for Hld bears no similarity to any obtained among the results described above.

\section{DISCUSSION}

As in the previous studies, all strains in the CXB set, including the progenitor strains, were able to improve performance with repeated training, but at different rates. There is a very strong association between strain and avoidance, but in some strains, acquisition (or performance) is very much a function of the time of day at which 
training and testing occur. For example, in the context of hurdling a low barrier to avoid impending shock to the feet, Strains CXBD, CXBE, and CXBH tend to exhibit more successful avoidance performance than most other strains of the CXB set, including the progenitors, in the early afternoon. However, the same procedure invoked late in the morning, while producing essentially similar performance by Strains CXBD and CXBE, reveals significantly inferior performance by Strain $\mathrm{CXBH}$. In other strains, TOD is essentially irrelevant, but the configuration of the barrier in the testing apparatus is of major importance. In some strains, these factors interact to a greater or lesser degree; in other strains, they do not interact at all. Thus, both apparatus configuration and TOD may significantly affect shuttlebox performance.

In the present instance, performance may be viewed most accurately as reflecting the influence of genetic determinants. Nevertheless, although one may indeed point to a gene (or to genes) as a major determinant of a behavior (in this case, two-way avoidance learning), it may be true only of certain genomes in certain contexts. As in the present case, responses to apparent circadian varia- tion and to environmental stimuli--responses that themselves may reflect major genetic influences-may supersede or mask such effects. One is able to observe only what occurs within the boundaries of imposed constraints, including constraints upon stimulus milieu and available responses. Generalization beyond these boundaries may be less than meaningful, if not misleading.

Is it because the behavioral differences in some instances or situations are overwhelmed, obscured by more powerful genetic determinants, that the genetic contributions to the observed behavior are identifiable under only some, and not all, conditions, or is it because, under some conditions, the relevant genes contribute, while under others, they are turned off (i.e., become inoperative)? Recent findings concerning the role of immediate early genes in learning and the availability of appropriate techniques make this question relevant and potentially amenable to investigation (Nikolaev, Kaminska, Tischmeyer, Matthies, \& Kaczmarek, 1992; Nikolaev, Werka, \& Kaczmarek, 1992).

Possible contributions of maternal factors (including nonnuclear inheritance) to shuttlebox performance in



Figure 4. Topography of shuttlebox avoidance-acquisition curves for C57BL/6ByJ, BALB/cByJ, and the CXB set of recombinant inbred strains with the slot partition. The leading (time of day, or TOD) surfaces of the performance curves are alternately darkly shaded or not shaded, to aid in distinguishing strains across the TOD dimension. The TODs are distinguished within strains by shading of the sessions surface. Vertical lines on a curve surface mark sessions; horizontal lines indicate mean number of avoidances, in 5-unit increments. 


\begin{tabular}{|c|c|c|c|}
\hline & $\begin{array}{l}\text { HURDLE } \\
\text { TOD } 1\end{array}$ & $\begin{array}{c}\text { HOLE } \\
\text { TOD } 1 \\
\text { JED B6 H IGK/C }\end{array}$ & $\begin{array}{c}\text { SLOT * } \\
\text { B6 J /CEDGKIH }\end{array}$ \\
\hline $\begin{array}{l}\text { Session } 1 \\
\text { Session } 2\end{array}$ & $\frac{B 6 / C K E \mid J H D G}{/ C J B 6 K E \mid G H D}$ & JIEDKB6G/CH & $\mathrm{J} / \mathrm{CGBGEDK}$ I H \\
\hline Session 3 & IC J BG I EGK K H & $\mathrm{J} I \mathrm{~K} / \mathrm{C} G \mathrm{D}$ B6 E H & KC G JEDK B6 I H \\
\hline Session 4 & Ic E B6 G J JKDH & $1 \mathrm{~J} / \mathrm{CG} D \mathrm{~B} G \mathrm{EKK} H$ & KU JGED $\overline{K I B 6} \mathrm{H}$ \\
\hline Session 5 & $E / C B 6 G \mid J H D K$ & $\mathrm{JG|/CB6DKEH}$ & $G / C J E D K / B 6 H$ \\
\hline Session 1 & $\begin{array}{c}\text { TOD } 2 \\
\text { /C B6 J IHKG DE } \\
\end{array}$ & $\begin{array}{c}\text { TOD } 2 \\
\mathrm{~J} I / C K H G D E B 6 \\
\end{array}$ & \\
\hline Session 2 & $\angle C J \mid B 6 H K G E D$ & $\mathrm{~J} \mid \mathrm{G} / \mathrm{CKH} B 6 \mathrm{DE}$ & \\
\hline Session 3 & $\not c J H B 6 \mid K G E D$ & J।/C GKH B6 DE & \\
\hline Session 4 & /C J H B6 I K GED & $1 \mathrm{~J} / \mathrm{CGHBKKDE}$ & \\
\hline Session 5 & $\frac{/ C \mathrm{~J} B G \mathrm{GH} \mid \mathrm{EK} D}{\text { TOD } 3}$ & $\frac{1 \mathrm{JG} / \mathrm{CH} \overline{\mathrm{BEKED}}}{\text { TOD } 3}$ & \\
\hline Session 1 & K/C B6 JDGE I H & E J B6 G I /CKHD & \\
\hline Session 2 & $\mathrm{JBG/CGKIDEH}$ & $K / C \mid G J D B G E H$ & \\
\hline Session 3 & $\underline{B G G / C J K I H E D}$ & $\angle C D G K J B 6 \mid E H$ & \\
\hline Session 4 & $K \mid G / C B 6 J E H D$ & $\angle K D G \mid J B G E H$ & \\
\hline Session 5 & GK/C B6 I JHED & ICKGDIJBGE H & \\
\hline
\end{tabular}

Figure 5. Strain distributions for each partition, Sessions 1-5, for each time of day (TOD). Strain designations are abbreviated as follows: Bb, C57BL/6ByJ; /c, BALB/cByJ; D, CXBD/ByJ; E, CXBE/ByJ; G, CXBG/ByJ; H, CXBH/ByJ; I, CXBI/ByJ; J, CXBJ/ByJ; K, CXBK/ByJ. Strains sharing an underline are not statistically significantly different $(\alpha=p>.05)$. *The strain $\times$ TOD interaction was not significant.

these strains remain to be examined. Isolation-induced fighting in the CXB set was investigated by Eleftheriou, Bailey, \& Denenberg (1974), who reported minimal effects of postnatal maternal behavior, but possible influence of cytoplasm of the ovum. Buselmaier, Vierling, Balzereit, \& Schwegler (1981), using several inbred mouse strains but not the CXB set, found that various environmental manipulations (i.e., fetal transplantation, crossfostering, isolation stress, and daily handling) had no measurable effect upon (jump-up) avoidance performance. In view of the current findings with respect to intratest variables, it would be advisable to assess the effect of such pretest manipulations upon CXB mice.

The present results, at all times of day and for all partitions, are more or less at variance with earlier findings (Oliverio et al., 1973; Peeler, 1987), particularly with respect to the performance of the BALB/c mice. There is, however, a rough correspondence. In the present study, averaged over the three TODs (see Figure 1), the distribution of strains for the hurdle configuration is similar, except for Strain BALB/c, and possibly Strain CXBI, to that reported by Peeler (1987). There were procedural differences between the hurdle portion of the present study and that of the earlier one (Peeler, 1987). The distribu- tion of practice (i.e., number of trials per session), the number of sessions, and the total number of trials were set in the present study to match the procedure of Oliverio et al. Nevertheless, performance over sessions, as discussed below, was very similar to that reported by Peeler (1987) for most strains. The one remaining procedural difference - control of time of day of testing, with sessions occurring throughout the day--appeared essentially to eliminate the discrepancies (except with respect to Strain BALB/c) between Peeler's (1987) study and the unpublished attempt at replication mentioned in the Introduction (Peeler, 1988). Presumably, the present study more closely resembled the 1987 study with respect to the distribution of testing over the 8-h work day. Thus, essentially, the results of the earlier study, except for one major deviation, are replicated.

As shown in Table 1, the distribution obtained by Oliverio et al. (1973) may be compared with the strain distribution obtained in the present study using the hole partition, the configuration most closely resembling that used by Oliverio et al. Although there are similarities (Strains CXBI and CXBJ have the lowest scores), the statistical dichotomy was not obtained, and, statistically and in terms of place, the relations among strains in the 
two distributions are quite dissimilar. Using the same barrier configuration, number of trials per session and total number of trials did not resolve the discrepancy between the findings of Oliverio et al. and those of Peeler (1987).

Other procedural differences exist between the present study and that of Oliverio et al. (1973), principally in terms of the CS, US, and ITI durations $(4,6$, and 16 sec vs. 5,25 , and $30 \mathrm{sec}$, respectively). Although these may contribute to differences between the 1973 and 1987 studies (a question for future investigation), they do not explain the drastically altered performance of the BALB/c mice in the present study. These parameters are identical to those used by Peeler (1987), in which BALB/c mice produced superior avoidance performance. Stimulus parameters have been noted to affect avoidance performance in mice (Royce, 1966, 1972), although this may not be related to initial response to electric shock (Wahlsten, 1972). The role of stimulus parameters in the discrepancy noted above requires further investigation.

In my earlier study (Peeler, 1987), TOD was not closely controlled, nor was it recorded. Similarly, Oliverio et al. (1973) mention no consideration of this variable. TOD in the present study, however, was a significant main effect in three strains (CXBE, CXBH, and CXBI); it interacted with partition in five additional strains. TOD thus may be a confounding factor leading to disparate results in the three studies. A comprehensive analysis of the activity rhythms and performance variations in these strains over a $24-\mathrm{h}$ period would be instructive, but circadian analyses are beyond the scope of the present study.

In the earlier study (Peeler, 1987), too, the subjects were tested for only 140 trials over 14 sessions. In the present study, it is noteworthy that for the most part, the interstrain differences were most pronounced at 150 trials (Session 3), after which, continued acquisition toward asymptote obscured many strain differences. Despite the different number of trials per session, the present data and those obtained by Peeler (1987) are similar in terms of the performance scores for most strains at a given number of trials. Thus the level of performance, as well as the relationships among strains, are largely consistent between the studies. Although there are significant differences in strain performance during the final session (Trials 201-250), it is perhaps more meaningful to compare performance either during acquisition (before performance reaches asymptote) or in terms of trials to criterion (i.e., rate of acquisition). Buselmaier et al. (1981) reached a similar conclusion. Although the acquisition curves of the present and the previous (Peeler, 1987) study are roughly comparable for the same number of trials, practice distribution and its possible interaction with genotype warrants further investigation.

Although Peeler (1987) and Oliverio et al. (1973) found Strain BALB/c to be superior to Strain C57BL/6, BALB/c mice have previously been noted to give inferior shockavoidance performance. Collins (1964) reported BALB/c mice to be the poorest performers of five inbred strains. Royce (1972) found BALB/c mice to be the slowest to acquire conditioning among nine strains. C57BL/6 mice were not among those tested in either of these studies. However, Schlesinger and Wimer (1967) found BALB/c mice to be inferior to C57BL/6 mice in a (nonshuttlebox) shock-avoidance task. Nevertheless, a greater number of studies have found BALB/c mice to be among the better performing strains, and C57BL/6 mice to be inferior. Few reports of avoidance conditioning include both $\mathrm{BALB} / \mathrm{cBy}$ and C57BL/6By mice.

As indicated in the Results section, there well may be strain-associated differences in response to stress and/or strategies to escape. Differences in response style to footshock in different mouse strains have been noted by others (Shanks \& Anisman, 1993; Takagi \& Makino, 1991; Tsutsumi \& Makino, 1990; Wahlsten, 1972). BALB/c mice have been noted to be particularly vulnerable, compared with C57BL/6 mice, to the impact of stressors (Hervé et al., 1979; Shanks \& Anisman, 1993; Shanks, Zalcman, Zacharko, \& Anisman, 1991), and there is increasing evidence that anxiety plays a dominant role in the early phase of shuttlebox avoidance (FernándezTeruel et al., 1991). Nevertheless, the differences between the present work and the earlier study (Peeler, 1987) in terms of the level of performance of Strain BALB/cByJ remain unexplained. A systematic analysis is required of strain (or subject) differences in response styles and the ways in which these styles may enhance or inhibit performance under various constraints of apparatus configuration and procedure.

It is difficult to interpret the progressively poorer acquisition in most strains when comparing performance in hurdle, hole, and slot configurations. The apparatus used by Peeler (1987) and in the first phase of the present study was obviously different from that used by Oliverio et al. (1973) and by Bovet et al. (1969) in at least one dimension - namely, in the configuration of the barrier dividing the compartments. This difference, in light of the very disparate performance of the $\mathrm{BALB} / \mathrm{c}$ mice in the initial experiment (hurdle barrier), led to the hypothesis that the BALB/c mice would perform much better with a barrier similar to that used by the earlier studies. Thus, naive mice from each strain were tested with the same paradigm, except that the hurdle was replaced by the hole barrier. When the BALB/c mice continued to produce relatively poor performance-because, it was thought, they were probably running "blindly" around the compartment and thus did not notice the hole in the barrierthe barrier was once again changed to provide an opening with no obstruction at the bottom (i.e., the slot). If they were desperately nosing their way along each wall and into the floor (and ignoring anything above floor level), it was thought that they would more readily detect the opening through the barrier with this configuration. However, they apparently did not; if anything, performance was worse, not only for $B A L B / c$ mice, but for most other strains as well. Although apparatus and procedural variables were designed to be unchanging (except for the barrier) in all phases, and no changes were detected, it is quite possible (particularly since data collection extended over many months) that some sort of 
progressive influence that biased results may have gone undetected. It is also possible that, given the extremely dim ambient lighting, a complicating factor with the hole and slot partitions may have been a lack of contrast between the opening and the surrounding barrier. It is also conceivable that the barriers themselves are responsible for the performance differences, as the data seem to indicate. $\mathrm{CXBH}$ and $\mathrm{C} 57 \mathrm{BL} / 6$ mice were striking exceptions to the general performance decrement; their mean scores were virtually unchanged over the three barrier configurations. The strong interactive association of genotype with configuration warrants further investigation.

Although there are instances in which the progenitor strains are statistically significantly different (i.e., slot configuration, Sessions 2, 3, and 5; hole, TOD 2, Session 2), they are few, and in only one case is there statistically a dichotomy of the RI strains clustering with the progenitors (hole configuration, TOD 2, Session 2). This might be expected by chance. Thus, one must conclude that under all of the testing conditions, avoidance behavior is influenced by two or more genetic determinantsthat is, that there appears to be no major single gene effect. In no instance does there appear to be any relationship between avoidance performance and the hippocampal lamination defect (Hld) reported by Nowakowski (1984) as having a single major gene determinant.

\section{REFERENCES}

BEAU, J. (1991). Activity rhythms in inbred mice: I. Genetic analysis with recombinant inbred strains. Behavior Genetics, 21, 117-129.

Bovet, D., Bovet-Nitti, F., \& Oliverio, A. (1966). Short and long term memory in two inbred strains of mice. Life Sciences, 5, 415-420.

Bovet, D., Bovet-Nitti, F., \& Oliverio, A. (1969). Genetic aspects of learning and memory in mice. Science, 163, 139-149.

Buselmaier, W., Vierling, T., Balzereit, W., \& Schwegler, $H$ (1981). Genetic analysis of avoidance learning by means of different psychological testing systems with inbred mice as model organisms. Psychological Research, 43, 317-333.

Collins, R. L. (1964, March 13). Inheritance of avoidance conditioning in mice: A diallel study. Science, 140, 1188-1190.

Eleftheriou, B. E., Bailey, D. W., \& DenEnBerg, V. H. (1974). Genetic analysis of fighting behavior in mice. Physiology \& Behavior, 13, 773-777.

Fernández-Teruel, A., Escorihuela, R. M., Núñez, J. F., Zapata, A., BolX, F., Salazar, W., \& Tobeña, A. (1991). The early acquisition of two-way (shuttle-box) avoidance as an anxiety-mediated behavior: Pharmacological validation. Brain Research Bulletin, 26, 173-176.

Hervé, D., Tassin, J. P., Barthelemy, C., Blanc, G., Lavielle, S., \& GLOWINSKI, J. (1979). Difference in the reactivity of the mesocortical dopaminergic neurons to stress in the BALB/c and $\mathrm{C} 57 \mathrm{BL} / 6$ mice. Life Sciences, 25, 1659-1664.

HostetTER, R. C. (1966). Time of day effects on learning and open field activity. Psychonomic Science, 5, 257-258.

LAssalle, J. M., \& Le PAPE, G. (1978). Locomotor activity of two inbred strains of mice in a seminatural and a breeding cage environment. Behavior Genetics, 8, 371-376.

MANLY, K. F. (1993). A Macintosh program for storage and analysis of experimental genetic mapping data. Mammalian Genome, 4, 303313.

MoIsseT, B. (1977). Factors contributing to the modulation of norepinephrine uptake by synaptosomes from mouse brain cortex. Brain Research, 121, 113-120.

Nikolaev, E., Kaminska, B., Tischmeyer, W., Matthies, H., \&
KACZMAREK, L. (1992). Induction of expression of genes encoding transcription factors in the rat brain elicited by behavioral training. Brain Research Bulletin, 28, 479-484.

Nikolaev, E., Werka, T., \& Kaczmarek, L. (1992). C-fos protooncogene expression in rat brain after long-term training of two-way active avoidance reaction. Behavioural Brain Research, 48, 91-94. NowaKowsKI, R. S. (1984). The mode of inheritance of a defect in lamination in the hippocampus of BALB/c mice. Journal of Neurogenetics, 1, 249-258.

Oliverio, A., Eleftheriou, B. E., \& Bailey, D. W. (1973). A gene influencing active avoidance performance in mice. Physiology \& Behavior, 11, 497-501.

Oliverio, A., \& MaLORNI, W. (1979). Wheel running and sleep in two strains of mice: Plasticity and rigidity in the expression of circadian rhythmicity. Brain Research, 163, 121-133.

PeELER, D. F. (1987). Active avoidance performance in genetically defined mice. Behavioral \& Neural Biology, 48, 83-89.

PEELER, D. F. (1988). Unpublished data.

Peeler, D. F. (1989). Apparatus configuration, circadian effects and hippocampal anatomy in genetically defined mice. Society for Neuroscience Abstracts, 15, 610 .

Peeler, D. F. (1990). Two measures of activity in genetically defined mice as a function of strain, time of day, and previous experience. Psychobiology, 18, 327-338.

PeELER, D. F. (1995). Genetic and environmental contributions to anxiety in mice. 1995 AAAS Anual Meeting, Abstracts, 145.

PoIREL, C. (1988). Rhythmometric analyses of circadian variations of grooming behavior in the mouse: Research perspectives in behavioral chronobiology. Journal of General Psychology, 115, 187-201.

Possidente, B., \& Birnbaum, S. (1979). Circadian rhythms for food and water consumption in the mouse, Mus musculus. Physiology \& Behavior, 22, 657-660.

ROYCE, J. R. (1966). Optimal stimulus parameters in avoidance conditioning of inbred strains of mice. Multivariate Behavioral Research, $1,209-217$.

RoycE, J. R. (1972). Avoidance conditioning in nine strains of inbred mice using optimal stimulus parameters. Behavior Genetics, 2, 107 . 110.

SCHLESINGER, K., \& WIMER, R. (1967). Genotype and conditioned avoidance learning in the mouse. Journal of Comparative \& Physiological Psychology, 63, 139-141.

SchWARTZ, W. J., \& Zimmerman, P. (1990). Circadian timekeeping in $\mathrm{BALB} / \mathrm{c}$ and C57BL/6 inbred mouse strains. Journal of Neuroscience, 10, 3685-3694.

Shanks, N., \& ANisman, H. (1993). Escape deficits induced by uncontrollable footshock in recombinant inbred strains of mice. Pharmacology, Biochemistry \& Behavior, 46, 511-517.

Shanks, N., Zalcman, S., Zacharko, R. M., \& Anisman, H. (1991). Alterations of central norepinephrine, dopamine and serotonin in several strains of mice following acute stressor exposure. Pharmacology, Biochemistry \& Behavior, 38, 69-75.

TAKAGI, H., \& MAKINO, J. (1991). [Sequential structure of behavior and its modification in inbred strains of mice in a passive avoidance situation]. Shinrigaku Kenkyu, 62, 286-293. (In Japanese, with English abstract)

Tsutsumi, K., \& MaKino, J. (1990). [Escape-avoidance learning in two strains of inbred mice when two types of response can be alternatively effective]. Shinrigaku Kenkyu, 61, 255-262. (In Japanese, with English abstract)

Vitaterna, M. H., King, D. P., Chang, A.-M., Kornhauser, J. M., Lowrey, P. L., McDonald, J. D., Dove, W. F., Pinto, L. H., Turek, F. W., \& TAKaHASHI, J. S. (1994, April 29). Mutagenesis and mapping of a mouse gene, Clock, essential for circadian behavior. Science, 264, 719-725.

WAHLSTEN, D. (1972). Phenotypic and genetic relations between initial response to electric shock and rate of avoidance learning in mice. Behavior Genetics, 2, 211-240.

(Manuscript received July 11, 1994; revision accepted for publication March 7, 1995.) 